\title{
Hemoblast Bellows
}

National Cancer Institute

\section{Source}

National Cancer Institute. Hemoblast Bellows. NCI Thesaurus. Code C128796.

A proprietary investig ational device that is in trials for cardiothoracic, abdominal, and orthopedic lower extremity surgeries as an adjunct to hemostasis when control of bleeding by conventional procedures is ineffective or impractical. 